\title{
ASPECTOS DA BIOLOGIA DE EUPLUSIA VIOLACEA (BLANCHARD) (HYMENOPTERA, APIDAE, EUGLOSSINI)
}

\author{
Rui Carlos Peruquetti ${ }^{1}$ \\ Lucio Antonio de Oliveira Campos ${ }^{1}$
}

\begin{abstract}
ASPECTS OF BIOLOGY OF EUPLUSIA VIOLACEA (BLANCHARD) (HYMENOPTERA, APIDAE, Euglossini). Nests of orchid bee Euplusia violacea (Blanchard, 1840) colleted in trap-nests and in the wood of a construction in Viçosa, Minas Gerais $\left(20^{\circ} 45^{\prime} 30^{\prime \prime} \mathrm{S}, 42^{\circ} 52^{\prime} 05^{\prime \prime} \mathrm{W}\right)$, Brazil, were studied under laboratory condictions. Some aspects of the biology of these bees were observed, such as sex-ratio, development time, longevity and others. Some aspects of the behavior of $E$. violacia were also studied.

KEY WORDS. Apoidea, Euglossini, Euplusia violacea, euglossine nest, behavior
\end{abstract}

A despeito do grande interesse despertado pelas abelhas da tribo Euglossini, poucos dados a respeito da biologia desses insetos são conhecidos. Uma revisão sobre o assunto é feita por ZUCCHI et al. (1969).

A maioria dos trabalhos realizados limitam-se ao levantamento de espécies com auxílio de iscas odoríficas que permitem coletar machos (BENNETTI 1972; BRAGA 1976; CAMPOS et al. 1989; REBÊLO \& GARÓFALO 1991, REBÊLO \& MOURE 1995), o que não possibilita grandes inferências a respeito dos aspectos da biologia dessas abelhas.

KIMSEY \& DRESSLER (1986) listam, aproximadamente, 160 espécies de Euglossini. Dessas, pouco mais de 15\% têm seus ninhos descritos (KIMSEY 1987). GARÓFALO (1994) descreve os principais hábitos de nidificação de Euglossini.

O gênero Euplusia Moure, 1943 [para KIMSEY (1979) este gênero é sinônimo de Eufriesea Cockerell, 1908] tem várias espécies sazonais e muitas delas são encontradas apenas durante a estação chuvosa, apresentando uma geração por ano (KIMSEY 1982, 1987; DrESSLER 1982; WITMANN et al. 1989). Constróem ninhos solitários, com algumas espécies, como E. auriceps (Friese, 1899), E. surinamensis (Linnaeus, 1758) e E. violacea, nidificando em agregados (SAKAGAMI \& MICHENER 1965; MYERS \& LOVELESS 1976; DRESSLER 1982; GARÓFALO \& SERRANO 1992).

Euplusia violacea é encontrada na região Sul e Sudeste do Brasil, além de Mato Grosso; chegando até o paralelo $45^{\circ} \mathrm{S}$ (região de Chubut na Argentina) (KIMSEY 1982). Em Viçosa, Minas Gerais (2045’30"S, 4252’05"W), essa espécie é ativa de meados de outubro até meados de março, aproximadamente 150 dias, sendo os machos atraídos por cineol ou vanilina (atrativos fortes), ou escatol (atrativo fraco). WITMANN et al. (1989) registraram machos dessa espécie visitando iscas odoríferas por 90 dias no Rio Grande do Sul.

1) Departamento de Biologia Geral, Universidade Federal de Viçosa. 36571-000 Viçosa, Minas Gerais, Brasil. 
A descoberta de um grande conjunto de ninhos de Euplusia violacea construídos no madeiramento de uma construção que seria derrubada, ofereceu oportunidade para o estudo de alguns aspectos da biologia dessa espécie.

\section{MATERIAL E MÉTODOS}

Os ninhos naturais foram construídos dentro de frestas e, ou, orifícios existentes no madeiramento que sustentava a cobertura de um barracão coberto de sapé no Campus da Universidade Federal de Viçosa. Em fevereiro de 1994 foram observadas fêmeas transportando material vegetal (ritidoma e resina) para construção e pólen para o aprovisionamento dos ninhos. Em 22 de dezembro de 1994, quando os adultos encontravam-se novamente ativos, colocou-se junto ao madeiramento, ninhos armadilhas constituídos de gomos de bambu, medindo aproximadamente $21,0 \mathrm{~cm}$ de comprimento e com orifícios variando de $1,0-1,5 \mathrm{~cm}$ de diâmetro, com uma das extremidades fechada pelo próprio nó, previamente abertos no sentido longitudinal, sendo as peças resultantes unidas com fita adesiva (fita "crepe"). Em 20 de fevereiro de 1995 foi observada uma fêmea transportando material para um desses ninhos armadilhas. Tanto os ninhos armadilhas, quanto os fundados no madeiramento, foram retirados do campo em 21 de abril de 1995, ocasião em que foi desmanchado o barracão. Alguns dos ninhos existentes no madeiramento foram abertos para observações gerais e todos foram mantidos em laboratório até a emergência dos adultos.

Dos ninhos abertos foram coletadas 25 larvas que foram postas em frascos tipo "penicilina" forrados com papel filtro e mantidos na posição horizontal até a emergência dos adultos.

Adultos que emergiram dos frascos e de ninhos intactos, foram mantidos em gaiolas de madeira e organza, com dimensões de $31,0 \times 31,0 \times 31,0 \mathrm{~cm}$, para observações de seu comportamento. Para a alimentação das abelhas foi oferecido xarope de mel e água (três partes de mel para uma de água) em recipiente aberto com algodão em seu interior. Machos e fêmeas foram mantidos juntos após a emergência.

Em algumas gaiolas foi colocado, pendurado no teto, um chumaço de algodão embebido em cineol ou solução alcóolica de vanilina para verificação da coleta dessas substâncias pelos machos em cativeiro.

A razão sexual foi calculada de acordo com a fórmula:

$\mathrm{n}^{\circ}$ de fềmeas

\section{RESULTADO E DISCUSSÃO}

$\overline{n^{\circ} \text { de fềmeas }+n^{\circ} \text { de machos }}$

Os resultados referentes à época de pupação, emergência e sexo dos indivíduos coletados nos ninhos estão sumariados na tabela I.

Nos machos, o tempo entre pupa e imago foi em média $43,8 \pm 4,25$ dias $(\mathrm{n}=12)$. Nas fêmeas $52,0 \pm 1,83$ dias $(\mathrm{n}=4)$, diferença estatisticamente significativa $(\mathrm{t}=3,71 ; \mathrm{P}=0,002)$. De um modo geral, os machos emergiram em meados de setembro e as fêmeas no início de outubro. Assim, os machos emergem antes das fêmeas. GARÓFALO \& SERRANO (1992) observaram que machos saiam do ninho antes das fêmeas e sugeriram que eles seriam produzidos nas últimas células construídas pelas fêmeas fundadoras. A observação dos ninhos construídos em 
ninhos armadilhas mostram que, de fato, os machos são produzidos nas últimas células construídas pelas fêmeas (Tab. II). O mesmo foi observado em Euplusia auriceps por GARÓFALO et al. (1993).

Tabela I. Data da pupação, da emergência e tempo de desenvolvimento de pupa a adulto de indivíduos obtidos a partir de larvas coletadas dos ninhos de Euplusia violacea mantidos em laboratório.

\begin{tabular}{ccccc}
\hline $\begin{array}{c}\text { Número } \\
\text { da larva }\end{array}$ & Sexo & $\begin{array}{r}\text { Data da pupação } \\
(1995)\end{array}$ & $\begin{array}{c}\text { Data da emergência } \\
(1995)\end{array}$ & $\begin{array}{c}\text { Tempo de } \\
\text { desenvolvimento pupal }\end{array}$ \\
\hline 1 & Macho & $6 / 7$ & $31 / 8$ & 55 \\
2 & Macho & $2 / 8$ & $15 / 9$ & 43 \\
3 & Macho & $4 / 8$ & $18 / 9$ & 44 \\
4 & Macho & $5 / 8$ & $18 / 9$ & 43 \\
5 & Macho & $6 / 8$ & $14 / 9$ & 39 \\
7 & Macho & $8 / 8$ & $19 / 9$ & 41 \\
8 & Macho & $8 / 8$ & $20 / 9$ & 42 \\
9 & Macho & $9 / 8$ & $22 / 9$ & 43 \\
12 & Macho & $10 / 8$ & $20 / 9$ & 48 \\
14 & Macho & $11 / 8$ & $29 / 9$ & 45 \\
15 & Macho & $11 / 8$ & $26 / 9$ & - \\
17 & Macho & $17 / 8$ & $29 / 9$ & 51 \\
\hline 6 & Fêmea & $8 / 8$ & -9 & 53 \\
10 & Fêmea & $9 / 8$ & $30 / 9$ & 54 \\
11 & Fêmea & $9 / 8$ & $2 / 10$ & 50 \\
13 & Fêmea & $11 / 8$ & $4 / 10$ & \\
16 & Fêmea & $16 / 8$ & $6 / 10$ & \\
\hline
\end{tabular}

Tabela II. Sexo das larvas de Euplusia violacea nas células de quatro ninhos armadilhas. A célula número 1 é a primeira a ser construída pela fêmea. (M) Macho, (F) fêmea, (L) larva.

\begin{tabular}{ccccccccccc}
\hline \multirow{2}{*}{ Ninho } & \multicolumn{10}{c}{ Célula } \\
\cline { 2 - 11 } & 1 & 2 & 3 & 4 & 5 & 6 & 7 & 8 & 9 & 10 \\
\hline 1 & $\mathrm{~F}$ & $\mathrm{~F}$ & $\mathrm{M}$ & $\mathrm{M}$ & $\mathrm{M}$ & $\mathrm{M}$ & $\mathrm{M}$ & $\mathrm{M}$ & $\mathrm{M}$ & $\mathrm{M}$ \\
2 & $\mathrm{~F}$ & $\mathrm{M}$ & $\mathrm{M}$ & $\mathrm{L}$ & $\mathrm{M}$ & & & & & \\
3 & $\mathrm{~F}$ & $\mathrm{M}$ & $\mathrm{M}$ & $\mathrm{M}$ & $\mathrm{M}$ & & & & & \\
4 & $\mathrm{~F}$ & $\mathrm{~F}$ & $\mathrm{M}$ & $\mathrm{M}$ & & & & & & \\
\hline
\end{tabular}

Do ninho armadilha que estava sendo aprovisionado em 20 de fevereiro de 1995 emergiu um macho em 08 de outubro de 1995, as demais larvas morreram. Com isso é possível estimar o tempo de desenvolvimento dos machos dessa espécie em, aproximadamente, 228 dias e o das fêmeas em 243 dias, visto que essas emergiram, aproximadamente, 15 dias após os machos. As larvas permanecem no último ínstar durante toda a estação seca, que em Viçosa se estende de maio a setembro (GOLFARI 1975).

No laboratório o tempo de vida dos machos adultos foi, em média, $23,6 \pm 1,85$ dias $(n=7)$. As fêmeas viveram $54,0 \pm 1,41$ dias $(n=2)$. Os dados obtidos monstram que as fêmeas vivem mais que os machos, como haviam sugerido ACKERMAN \& Montalvo (1985). 
A partir dos ninhos coletados foram obtidos 131 machos e 59 fêmeas, resultando em uma razão sexual de 0,31.

Grande parte dos trabalhos com Euglossini se baseiam em dados obtidos a partir de machos coletados com auxílio de iscas odoriferas, assim o conhecimento da razão sexual das espécies da tribo é bastante importante para análises de estrutura populacional nessas abelhas. E. violacea, nesse estudo, mostrou produzir mais machos do que fêmeas, indicando que nessa espécie os machos são um bom parâmetro para estimativas populacionais. A razão sexual encontrada em Euglossini por outros autores, apesar de serem poucos os dados, encontra-se na tabela III.

Tabela III. Razão sexual em Euglossini, dados da literatura. (A) Agregado, (N) ninho, (M) macho, (F) fêmea.

\begin{tabular}{|c|c|c|c|}
\hline Espécie & & zão sexual & Autor \\
\hline Eulaema cingulata Fabricius, 1804 & 0,86 & $(1 \mathrm{~N}, 125 \mathrm{~F}, 20 \mathrm{M})$ & DODSON (1966) \\
\hline Eulaema meriana (Oliver), 1789 & 0,43 & $(1 \mathrm{~N}, 3 \mathrm{~F}, 4 \mathrm{M})$ & ACKERMAN \& MONTALVO (1985) \\
\hline Eulaema nigrita Lepeletier, 1814 & 0,83 & $(1 \mathrm{~N}, 20 \mathrm{~F}, 4 \mathrm{M})$ & ZUCCHI et al. (1969) \\
\hline Eulaema nigrita Lepeletier, 1814 & 0,76 & $(2 \mathrm{~N}, 28 \mathrm{~F}, 9 \mathrm{M})$ & SANTOS \& GARÓFALO (1994) \\
\hline Eulaema nigrita Lepeletier, 1814 & 0,86 & $(1 \mathrm{~N}, 25 \mathrm{~F}, 4 \mathrm{M})$ & PEREIRA-MARTINS \& KERR (1991) \\
\hline Eulaema nigrita Lepeletier, 1814 & 0,27 & $(2 \mathrm{~N}, 16 \mathrm{~F}, 43 \mathrm{M})$ & ACKERMAN \& MONTALVO (1985) \\
\hline Eulaema terminata Smith, 1874 & - & $(1 \mathrm{~N}, \mathrm{OF}, 9 \mathrm{M})$ & BENNETT (1965) \\
\hline Exaerete frontalis (Guérin), 1845 & $0,50^{*}$ & $(1 \mathrm{~N}, 11 \mathrm{~F}, 11 \mathrm{M})$ & ACKERMAN \& MONTALVO (1985) \\
\hline Eufriesea violacea (Blanchard, 1840) & 0,38 & $(1 \mathrm{~A}, 9 \mathrm{~F}, 15 \mathrm{M})$ & GAROFALO \& SERRANO (1992) \\
\hline Eufriesea auriceps (Friese), 1899 & 0,39 & $(10 N, 13 F, 20 M)$ & GAROFALO et al. (1993) \\
\hline Euglossa cordata (Linnaeus, 1758) & 0,45 & $(13 \mathrm{~N}, 24 \mathrm{~F}, 29 \mathrm{M})$ & GARÓFALO (1992) \\
\hline Euglossa ignita Smith, 1874 & 0,50 & $(21 \mathrm{~N})$ & DODSON (1966) \\
\hline Euglossa aff melanotricha Moure, 1967 & - & $(1 \mathrm{~N}, 2 \mathrm{~F}, 0 \mathrm{M})$ & GAROFALO et al. (1993) \\
\hline Euglossa melanotricha Moure, 1967 & 0,36 & $(1 \mathrm{~N}, 3 \mathrm{~F}, 5 \mathrm{M})$ & SAKAGAMI et al. (1967) \\
\hline Euglossa pleosticta Dressler, 1985 & 0,35 & $(5 \mathrm{~N}, 6 \mathrm{~F}, 11 \mathrm{M})$ & GAROFALO et al. (1993) \\
\hline Euglossa townsendi Cockerell, 1904 & 0,58 & $(3 N, 7 F, 5 M)$ & GAROFALO et al. (1993) \\
\hline Euglossa truncata Rebélo \& Moure, 1995 & 0,20 & $(7 \mathrm{~N}, 6 \mathrm{~F}, 24 \mathrm{M})$ & GAROFALO et al. (1993) \\
\hline Euglossa turbiniflex Dressler, 1978 & 0,63 & $(1 \mathrm{~N}, 5 \mathrm{~F}, 3 \mathrm{M})$ & YOUNG (1985) \\
\hline Euglossa sp. & - & $(1 \mathrm{~N}, 4 \mathrm{~F}, 0 \mathrm{M})$ & YOUNG (1986) \\
\hline
\end{tabular}

*. Adultos que emergiram de ninho de Eulaema meriana

Foi observado que tanto machos como fêmeas de E. violacea, após a última muda, permanecem 2-3 dias na célula de cria. Quando saem, apenas as fêmeas retornam ao ninho, passando a noite em seu interior. Esse comportamento se manteve por 4 dias, após os quais, nas condições de laboratório, abandonaram o ninho e passaram a se agrupar com os machos. Estes grupos se tornavam mais compactos durante a noite, mas persistiam durante várias horas do dia. Indivíduos de ambos os sexos, quando em grupo, prendiam-se pelas mandíbulas na tela (organza) ou no madeiramento da gaiola, permanecendo o resto do corpo livre.

Machos e fêmeas quando deixam as células de cria repousam por algum tempo presos pelas mandíbulas, e defecam sobre suas patas posteriores, espalhando o excremento por todo o corpo com auxílio das outras patas. Este comportamento é abandonado pelas fêmeas após 3-4 dias, persistindo nos machos. Em certa ocasião foi observado um macho depositando este excremento em suas tíbias posteriores. 
As iscas odoríferas colocadas nas gaiolas (cineol ou vanilina) não foram visitadas pelos machos em momento algum durante as observações. Entretanto foi comum observar que, quando se colocava cineol na gaiola, os machos se agitavam, sendo encontrados, posteriormente, vários deles mortos nestas gaiolas. Nas que/se colocava vanilina não havia qualquer modificação do comportamento ou aumento da mortalidade dos machos. ACKERMAN \& MONTALVO (1985) também observaram que os machos de Euglossini podem ser mantidos em cativeiro sem acesso à fragrâncias florais.

Por várias vezes observou-se machos e fêmeas posicionados frente a frente por longo tempo. Neste período, caso o macho se movimentasse, a fêmea levantava uma ou as duas patas posteriores; não foi possível determinar, a razão desse comportamento. Não foram observadas cópulas entre os indivíduos mantidos em gaiolas para observação. Uma fêmea limpou uma célula de cria e a estava aprovisionando com o mel oferecido para alimentação das abelhas. Esta fêmea foi dissecada e não foram encontrados espermatozóides em sua espermateca.

Quando machos recém emergidos eram postos em gaiolas diferentes daquela em que haviam nascido, estes ficavam imóveis e emitiam um zumbido que durava aproximadamente 15 segundos, após isto movimentavam-se, ora voando, ora apenas caminhando. Este comportamento foi observado apenas em machos colocados em gaiolas onde já haviam outras abelhas. Estas não apresentaram nenhuma reação aparente nessas ocasiões.

Associados aos ninhos foram encontrados Melilitobia sp (Hymenoptera, Eulophidae), três ninhos com várias células parasitadas, e meloídeos (Coleoptera) do gênero Meloetyphlus Walterhouse, 1972, dois ninhos com a penúltima célula construída pela fêmea ocupada por esse parasita.

Como todas as observações foram realizadas com abelhas que emergiram em cativeiro e aí permaneceram e visto, também, as reduzidas dimensões das gaiolas para observação, parte do comportamento documentado pode ter sido influenciado por estas condições. Trabalhos de observação comportamental, conduzidos a partir de indivíduos recém emergidos, e realizados sob condições controladas em ambientes amplos, podem contribuir para o melhor entendimento do comportamento e das relações que estas abelhas mantém entre si e com o ambiente.

\section{CONCLUSÕES}

Machos de E. violacea apresentaram tempo de desenvolvimento de aproximadamente 228 dias e fêmeas de 243 dias.

A razão sexual encontrada foi de 0,31 . Em cada ninho a abelha põe óvulos fecundados que produzirão fêmeas nas primeiras células que constróem, nas últimas põe óvulos não fecundados que se desenvolvem em machos.

Em condições de cativeiro as fêmeas vivem mais que os machos. Nas mesmas condições não foram observadas cópulas e machos não foram observados coletando substâncias odoríferas, a eles oferecidas (cineol ou vanilina) como o fazem na natureza. 
AGRADECIMENTOS. Os autores agradecem aos funcionários da Prefeitura do Campus da Universidade Federal de Viçosa pela colaboração, ao Prof. José Lino Neto pela dissecção das abelhas, ao CNPq e FAPEMIG pelo auxilio financeiro e aos consultores anônimos da Revista Brasileira de Zoologia pelas sugestões apresentadas.

\section{REFERÊNCIAS BIBLIOGRÁFICAS}

ACKerman, J.D. \& A.M. Montalvo. 1985. Longevity of euglossine bees. Biotropica 17 (1): 79-81.

BENNETT, F.D. 1965; Notes on a nest of Eulaema terminata Smith (Hymenoptera, Apoidea) with a suggestion of the occurence of a primitive social system. Insectes Sociaux 12 (1): 81-92.

1972. Baited McPhail fruitfly traps to collect euglossine bees. Jour. New York Entomol. Soc. 80: 137-145.

BRAGA, P.I.S. 1976. Atração de abelhas polinizadoras de Orchidaceae com auxílio de iscas-odores na campina, campinara e floresta tropical úmida da região de Manaus. Ciência e Cultura 28 (7): 767-773.

Campos, L.A. DE O.; F.A. DA Silveira; M.L. DE Oliveira; C.V.M. AbRANTES; E.F. MORATO \& G.A.R. DE MELO. 1989. Utilização de armadilhas para a captura de machos de Euglossini (Hymenoptera, Apoidea). Revta bras. Zool. 6 (4): 621-626.

DoDson, C.H. 1966. Ethology of some bees of the tribe Euglossini (Hymenoptera: Apidae). Jour. Kansas Entomol. Soc. 39 (4): 607-629.

DRESSLER, R.L. 1982. Biology of the orchid bees (Euglossini). Ann. Rev. Ecol. Syst. 13: 373-394.

GARÓFALO, C.A. 1992. Comportamento de nidificação e estrutura de ninhos de Euglossa cordata (Hymenoptera: Apidae: Euglossini). Rev. Brasil. Biol. 52 (1): 187-198.

. 1994. Biologia de nidificação dos Euglossinae (Hymenoptera, Apidae).

- Anais I Encontro sobre Abelhas, Ribeirão Preto, 1: 17-26.

Garófalo, C.A. \& J.C. SerRano. 1992. Reutilização do ninho por Eufrisea violacea (Hymenoptera, Apidae, Euglossini). Naturalia, São Paulo, (no. especial): 234.

Garófalo, C.A.; E. CAMILlO; J.C. SerRano \& J.M.M. RebÊLo. 1993. Utilization of traps nests by Euglossini species (Hymenoptera: Apidae). Rev. Brasil. Biol. 53 (2): 177-187.

Golfari. L. 1975. Zoneamento ecológico do estado de Minas Gerais para reflorestamento. Belo Horizonte, PRODEPEF, PNUB, FAO, IBDF, BRA-45, $65 \mathrm{p}$.

KIMSEY, L.S. 1979. Synonymy of the genus Euplusia Moure under Eufriesea Cockerell (Hymenoptera, Apidae, Euglossini). Pan-Pacific Entomologist 55 (2): 126.

KIMSEY, L.S. 1982. Systematics of bees of the genus Eufriesea (Hymenoptera, Apidae). Univ. California Publ. Entom. 95: 1-125. 
1987. Generic relationships within Euglossini (Hymenoptera: Apidae). Systematic Entomology 12: 63-72.

KIMSEY, L.S. \& R.L. DRESSLER. 1986. Synonymic species list of Euglossini. Pan-Pacifc Entomologist 62 (3): 229-36.

MYERS, J. \& M.D. LOVELESS. 1976. Nestin aggregations of the euglossine bee Euplusia surinamensis (Hymenoptera: Apidae): individual interations and the advantage of living together. Can. Ent. 108: 1-6.

Pereira-Martins, S.R. \& W.E. KerR. 1991; Biologia de Eulaema nigrita. 1. Construção de células, oviposição e desenvolvimento. Pap. Avuls Zool., São Paulo, 37 (13): 227-35.

REBÊLO, J.M.M. \& C.A. GARÓFALO. 1991. Diversidade e sazonalidade de machos de Euglossini (Hymenoptera, Apidae) e preferências por iscas-odores em um fragmento de floresta no sudeste do Brasil. Rev. Brasil. Biol. 51 (4): 787-799.

RebÊLo, J.M.M. \& J.S. Moure. 1995. As espécies de Euglossa Latreille do Nordeste de São Paulo (Apidae, Euglossinae). Revta bras. Zool. 12 (3): 445-466.

SAKAGAMI, F. \& C.D. MiChENER. 1965. Notes on the nests of two euglossine bees, Euplusia violacea and Eulaema cingulata (Hymenoptera, Apidae). Ann. Zool. Japon. 38 (4): 216-222.

SAKAGAMI, F.; S. LAROCA \& J.S. MourE. 1967. Two brazilian apid nest worth recording in reference to comparative bee sociology, with description of Euglossa melanotricha Moure sp.n. (Hymenoptera, Apidae). Ann. Zool. Japon. 40 (1): 45-54.

SANTOS, M.L. \& C.A. Garófalo. 1994; Arquitetura de ninhos e duração do período de aprovisionamento de células de macho e fêmea de Eulaema nigrita (Hymenoptera, Apidae, Euglossini). Anais I ESARP-FFCLRP, USP, Ribeirão Preto, 1: 161-70.

Wittmann, D.; R. Radtke; M. Hoffmann \& B. Blochtein. 1989. Seasonality and seasonal changes in preference for scent baits in Euplusia violacea in Rio Grande do Sul/Brasil (Hymenoptera: Apidae: Euglossini). Entomol. Gener. 14 (3-4): 217-221.

YouNG, A.M. 1985. Notes on the nest structure and emergence of Euglossa turbiniflex Dressler (Hymenoptera: Apidae: Bombinae: Euglossini) in Costa Rica. Jour. Kansas Entomol. Soc. 58 (3): 538-543.

1986; Presence of an orchid bee (Euglossa sp.) nest an an ant (Crematogaster limata palans) nest in a cacao pod (Theobroma cacao) (Hymenoptera: Apidae, Formicidae, Resp.). Entomo. News 97 (4): 156-62.

ZUCCHI, R.; S.F. SAKAGAMI \& J.M.F. CAMARGO. 1969. Biological observations on a Neotropical parassocial bee, Eulaema nigrita, with a review on the biology of Euglossinae (Hymenoptera, Apidae). A compartive estudy. Jour. Fac. Sc. Hokkaido Univ., Zoology, 17 (6): 271-380.

Recebido em 26.II.1996; aceito em 20.III.1997. 